\title{
Improved Dosing and Administration of Rivaroxaban when Prescribed by a Cardiologist
}

\author{
Madeline R Leiter, ${ }^{1}$ Kathleen A Packard, ${ }^{1,2}$ Yongyue Qi, ${ }^{1}$ Steven K Krueger ${ }^{2}$ \\ 1. Creighton University, School of Pharmacy and Health Professions, Omaha, NE, USA; 2. Bryan Health Medical Center, Bryan Heart Institute, \\ Lincoln, NE, USA
}

$\mathrm{R}$ ivaroxaban is a direct oral anticoagulant (DOAC) indicated to reduce risk of stroke and systemic embolism in patients with non-valvular atrial fibrillation (AF). A discrepancy exists between the recommended dosage and real-world use of DOACs, especially rivaroxaban, thus putting patients at risk of thromboembolic events. Methods: This retrospective study assessed real-world prescribing and patient adherence to dietary requirements during use of rivaroxaban in 116 patients with AF. Associations between prescriber specialty and the correct dosing and administration were assessed using the Chi-Square test. Results: Most rivaroxaban prescriptions were ordered by cardiologists (50.9\%). Sixty-nine patients (59.5\%) were taking the right dose at the correct time with an adequate meal. Of the 47 (40.5\%) taking rivaroxaban incorrectly, 39 (33.6\%) had not been administered an adequate meal and eight (6.9\%) were not prescribed the correct dose. Compared with other prescribers, patients were most likely to be taking the correct dose and administration when prescribed by cardiologists (72.9\% versus $45.6 \% ; p=0.003)$. Patients were least likely to be taking the correct dose and administration when prescribed by primary care providers ( $44.4 \%$ versus $69.0 \% ; \mathrm{p}=0.009$ ). This difference was driven by patients who did not take the treatment with an adequate meal. Conclusion: Inappropriate prescribing, administration and non-adherence to DOACs can have devastating consequences. This highlights the importance of formal systematic education of patients prescribed DOACs across the whole health system. Future studies are warranted to explore the impact of non-adherence to rivaroxaban dietary requirements on clinical outcomes.

\section{Keywords}

Anticoagulation, atrial fibrillation, cardiology

Disclosure: Steven K Krueger is on the Speaker's Bureau for Amgen, Boehringer Ingelheim, Novartis, Regeneron Pharmaceuticals, Sanofi, Orexigen Therapeutics, Janssen, Kowa Pharmaceuticals, Pfizer, Bristol Myers Squibb and Amarin. Madeline R Leiter, Kathleen A Packard and Yongyue Qi have no conflicts of interest to declare.

Review Process: Double-blind peer review.

Compliance with Ethics: All procedures were followed in accordance with the responsible committee on human experimentation and with the Helsinki Declaration of 1975 and subsequent revisions.

Authorship: All named authors meet the criteria of the International Committee of Medical Journal Editors for authorship for this manuscript, take responsibility for the integrity of the work as a whole and have given final approval for the version to be published.

open Access Statement: This article is published under the Creative Commons Attribution Noncommercial License, which permits any non-commercial use, distribution, adaptation and reproduction provided the original author(s) and source are given appropriate credit. (c) The Authors 2018.

Received: 28 September 2018

Accepted: 4 October 2018

Citation: Heart International. 2019;13(1):24-7

Corresponding Author: Kathleen Packard, School of Pharmacy and Health Professions, Creighton University, 2500 California Plaza, Omaha, NE, 68178, US.

E: KatiePackard@creighton.edu

Support: No funding was received in

the publication of this article.
Rivaroxaban is a direct factor Xa inhibitor indicated to reduce the risk of stroke and systemic embolism in patients with non-valvular atrial fibrillation (AF), treatment of deep vein thrombosis (DVT) and pulmonary embolism (PE). Furthermore, it is prescribed for risk reduction and prophylaxis for DVT and PE following hip or knee replacement surgery and is one of the most commonly prescribed direct oral anticoagulants (DOACS).

A substantial discrepancy exists between the recommended dosage and administration and real-world use of DOACs, putting patients at risk of serious thromboembolic events. ${ }^{2-4}$ Patients taking rivaroxaban are uniquely susceptible to administration errors due to dose-dependent requirements for co-administration with meals. As rivaroxaban has dose-dependent bioavailability, concomitant food recommendations vary with the dose. ${ }^{1,5}$ The bioavailability of the $10 \mathrm{mg}$ dose is estimated to be $80-100 \%$ and is not affected by food, whereas the bioavailability of the $20 \mathrm{mg}$ dose in the fasted state is approximately $66 \%$. Food increases the bioavailability of the rivaroxaban $20 \mathrm{mg}$ dose (mean area under the curve $[\mathrm{AUC}]$ and maximum concentration $\left[\mathrm{C}_{\max }\right.$ increasing by $39 \%$ and $76 \%$, respectively) and also decreases inter-individual variability, which ultimately improves the predictability of the pharmacokinetics. ${ }^{6}$ Kubitza et al. determined that the fat content of the meal was less important on the bioavailability of rivaroxaban, as they found no differences between a high-fat, high-calorie meal versus a high-carbohydrate meal. ${ }^{\top}$ Thus, taking rivaroxaban in a fasting state or with a small meal could reduce bioavailability and potentially increase the risk of thromboembolic event. The clinical implications of administering rivaroxaban with inconsistent meals or in the fasting state was highlighted in a case report of an individual that experienced recurrent $\mathrm{PE}$ due to irregular intake of meals during shift work?

The recommended dose of rivaroxaban in patients with AF and normal renal function is $20 \mathrm{mg}$ orally, once daily with the evening meal, ${ }^{1}$ which is how the drug was administered in the pivotal, phase III Rivaroxaban Once Daily Oral Direct Factor Xa Inhibition Compared with Vitamin $\mathrm{K}$ Antagonism for Prevention of Stroke and Embolism in Atrial Fibrillation (ROCKET AF) study.1.8 However, it does not appear that adherence to a full meal nor the macronutrient content of such meal was captured in ROCKET AF. ${ }^{8}$ The dose for patients with a creatinine clearance $15-50 \mathrm{ml} / \mathrm{min}$ is $15 \mathrm{mg}$. 'Dosages for treatment of DVT, PE or reduction in risk of recurrent venous thromboembolisms are $15 \mathrm{mg}$ orally twice daily with food for the first 21 days, followed by $20 \mathrm{mg}$ once daily with food. Dosages for prevention of DVT after hip or knee replacement surgery is $10 \mathrm{mg}$ once daily with or without food.' 
In addition to errors in administration with appropriate meals, rivaroxaban is not always dosed correctly, especially in patients with renal dysfunction. A recent analysis assessed DOAC dosing patterns and associated outcomes in 14,865 patients with AF and renal dysfunction. ${ }^{3}$ In patients with a renal indication for a reduced dose of rivaroxaban, $41.3 \%$ received standard doses. In patients with no renal indication for dose reduction, $15.1 \%$ of patients were underdosed. In those patients with a renal indication for dose reduction, the event rate per 100 person-years of ischaemic stroke or systemic embolism was 1.18 (0.26-11.45) for those on the reduced dose and 2.84 (1.19-8.47) for those on standard dosing. In the same cohort, the rate of major bleeding was 5.88 (3.19-11.94) for those on reduced dosing and 10.98 (7.01-18.05) for those on standard dosing, suggesting that overdosing nearly doubled the risk of bleeding without similar reduction in the risk of stroke. An increased risk of cerebral vascular accident was found in patients with normal renal function that were 'underdosed' in apixaban-treated patients, but not dabigatran or rivaroxaban. ${ }^{3}$ Not only can inappropriate prescribing affect clinical outcomes but patient adherence can also be detrimental. In another recent study, it was found that non-persistence with both rivaroxaban and dabigatran was associated with increased risk of cerebral vascular accident and or death. ${ }^{4}$

This retrospective study documents provider prescribing and real-world patient adherence to dietary requirements during self-administration of rivaroxaban for AF.

\section{Methods}

This retrospective study was conducted at Bryan Health Medical Center in Lincoln, NE between January 2014 and January 2017 in 116 consecutive, unique individuals. The protocol was reviewed and deemed exempt by the Creighton University Institutional Review Board. Patients were included if they were seen in cardiology consultation for AF and were already taking rivaroxaban prior to evaluation.

Data collected from each patient included prescribed dose, renal function (current and at the time of rivaroxaban initiation), prescriber specialty and patient adherence. Prescriber specialty was the specialty of the provider that initiated the prescription prior to the cardiology consult. Renal function was calculated using the Cockcroft-Gault equation using actual body weight. If the patient's actual body weight was greater than $20 \%$ of ideal, then adjusted body weight was used. A patient was considered dosed inappropriately using their renal function at the time of prescribing. Patients were considered non-adherent if they reported missing any doses, if they were administering during a fasting state or administering only with a small meal or snack. All patient adherence data was self-reported. The same investigator, a cardiologist, assessed whether or not the patient was taking with an adequate meal by asking the patient if they were taking with a meal, which meal and what the meal consisted of.

Categorical variables are presented as frequency and percent. Associations between professional specialty and the correct dosing and administration were assessed using the Chi-Square test. All statistical analysis was performed in IBM SPSS Statistics for Windows, Version 24.0 (Armonk, NY: IBM Corp.).

\section{Results}

Figure 1 indicates that the majority of prescriptions for rivaroxaban in this sample were ordered by cardiologists $(59,50.9 \%)$, followed by primary care physicians ([PCP] 45, 38.8\%), oncologists (4,3.4\%), neurologists $(3,2.6 \%)$, vascular surgeons $(3,2.6 \%)$ and general surgeons $(2,1.7 \%)$.

\section{Figure 1: Prescribers of rivaroxaban}

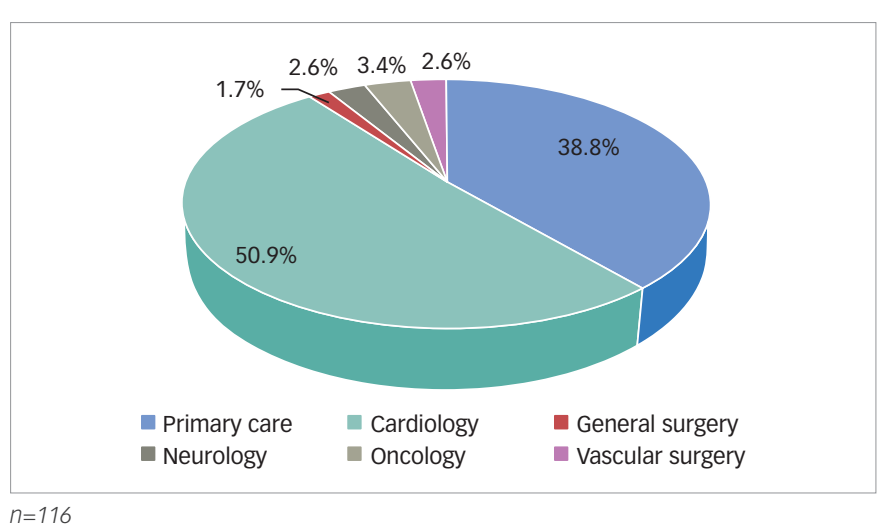

Table 1: Rivaroxaban dosing assessment

\begin{tabular}{|l|l|}
\hline Rivaroxaban prescribing pattern & $\begin{array}{l}\text { Number of patients } \\
\text { (percentage of whole) }\end{array}$ \\
\hline Everything correct & $69(59.5 \%)$ \\
\hline Incorrect & $47(40.5 \%)$ \\
\hline Not with a meal containing adequate fat & $39(33.6 \%)$ \\
\hline Wrong dose & $8(6.9 \%)$ \\
\hline Dose too high & $2(1.7 \%)$ \\
\hline Dose too low & $6(5.2 \%)$ \\
\hline$n=116$ & \\
\hline
\end{tabular}

Table 2: Comparison of prescribers by professional specialty

\begin{tabular}{|c|c|}
\hline Rivaroxaban prescribing pattern & $\begin{array}{l}\text { Number of patients } \\
\text { (percentage of whole) }\end{array}$ \\
\hline \multicolumn{2}{|l|}{ Cardiologist versus all other prescribers $(p=0.003)$} \\
\hline Cardiologist - correct (of 59 total cardiology patients) & $43(72.9 \%)$ \\
\hline $\begin{array}{l}\text { Cardiologist - incorrect (of } 59 \text { total cardiology } \\
\text { patients) }\end{array}$ & $16(27.1 \%)$ \\
\hline $\begin{array}{l}\text { All others correct (of } 57 \text { total other non-cardiology } \\
\text { prescriber patients) }\end{array}$ & $26(45.6 \%)$ \\
\hline $\begin{array}{l}\text { All others incorrect (of } 57 \text { total other non-cardiology } \\
\text { prescriber patients) }\end{array}$ & $31(54.4 \%)$ \\
\hline \multicolumn{2}{|l|}{ PCP versus all other prescribers $(p=0.009)$} \\
\hline PCP - correct (of 45 total PCP patients) & $20(44.4 \%)$ \\
\hline PCP - incorrect (of 45 total PCP patients) & $25(55.6 \%)$ \\
\hline $\begin{array}{l}\text { All others correct (of } 71 \text { total other non-PCP } \\
\text { prescriber patients) }\end{array}$ & $49(69.0 \%)$ \\
\hline $\begin{array}{l}\text { All others incorrect (of } 71 \text { total other non-PCP } \\
\text { prescriber patients) }\end{array}$ & $22(31.0 \%)$ \\
\hline \multicolumn{2}{|l|}{ PCP versus cardiologist $(\mathrm{p}=0.003)$} \\
\hline PCP - correct (of 45 total PCP patients) & 20 (44.4\%) \\
\hline PCP - incorrect (of 45 total PCP patients) & 25 (55.6\%) \\
\hline Cardiologist - correct (of 59 total cardiology patients) & $43(72.9 \%)$ \\
\hline $\begin{array}{l}\text { Cardiologist - incorrect (of } 59 \text { total cardiology } \\
\text { patients) }\end{array}$ & $16(27.1 \%)$ \\
\hline
\end{tabular}

$P C P=$ primary care physician

Of the 116 patients assessed, 69 (59.5\%) were taking the correct dose based on indication and renal function and taking at the correct time with an adequate meal (see Table 1). Of the 47 (40.5\%) taking rivaroxaban incorrectly, 39 (33.6\%) were not administered with an adequate meal and 
Table 3: Comparison of incorrect rivaroxaban dosing by professional specialty

\begin{tabular}{|l|l|}
\hline Rivaroxaban prescribing pattern & Number of patients (percentage of whole) \\
\hline Cardiologist & $n=16$ of 59 incorrectly dosed (27.1\%) \\
\hline Not administered with adequate meal (of 59 total cardiology patients) & $15(25.4 \%)$ \\
\hline Incorrect dose (of 59 total cardiology patients) & $1(1.7 \%)$ \\
\hline PCP & $n=25$ of 45 incorrectly dosed (55.6\%) \\
\hline Not administered with adequate meal (of 45 total PCP patients) & $21(46.7 \%)$ \\
\hline Incorrect dose (of total 45 PCP patients) & $4(8.9 \%)$ \\
\hline$P C P=$ primary care physician & \\
\hline
\end{tabular}

8 (6.9\%) were not prescribed the correct dose. Of the incorrectly dosed patients, 2 (1.7\%) were too high and $6(5.2 \%)$ were too low. All patients reported that they had not missed any doses.

Table 2 compares the prescribing pattern of rivaroxaban by professional specialty. Compared with all other prescribers, patients were most likely to be taking the correct dose with correct administration when prescribed by cardiologists $(72.9 \%$ correct versus $45.6 \%$ correct, respectively; $\mathrm{p}=0.003)$. Patients were least likely to be taking the correct dose with correct administration when prescribed by PCP (44.4\% correct versus $69.0 \%$ correct, respectively; $p=0.009$ ). It should be noted that of the 25 patients that were prescribed rivaroxaban by a PCP and taking it incorrectly, only 4 (8.9\%) were dosed incorrectly by the prescriber (see Table 3). However, 21 (46.7\%) of patients taking rivaroxaban prescribed by a PCP were not administered with an adequate meal.

\section{Discussion}

Inappropriate prescribing, non-adherence and inappropriate administration of anticoagulation can have devastating consequences. This study demonstrated a gap in patient awareness of proper administration of rivaroxaban with an adequate meal in that $33.6 \%$ of patients were not taking with a meal. This is similar to the $32.3 \%$ recently reported by Tellor et al. ${ }^{9}$ During this process, many patients anecdotally reported no knowledge of the requirement to take with an adequate meal. Patients under a cardiologist's care were most likely to be taking rivaroxaban correctly and this may be due to increased exposure of this specialty to continuing education regarding prescribing of DOACs. Alternatively, the advantage may be due to a team-based approach to care often utilised in cardiology practice groups. Both inpatient and outpatient care of AF patients is often multidisciplinary, allowing for collaborative and shared responsibility for patient education amongst cardiologists, mid-level providers, nurses and pharmacists.

Conversely, of the 45 patients prescribed rivaroxaban by a PCP, 21 (46.7\%) were not administered with an adequate meal. These results highlight the opportunity for community pharmacists to play a role in closing the gap in patient education as it relates to the dosing and administration of rivaroxaban. Community pharmacists are often the last healthcare providers in contact with the patient before outpatient medication administration. Survey data to assess the rivaroxaban patient education practices from various providers and also community pharmacists are warranted to better explain these findings.

Of the eight (6.9\%) patients prescribed the incorrect rivaroxaban dose, the majority (six patients) were underdosed. While underdosing has the potential to increase risk of cerebral vascular accident, overdosing appears to have nearly double the adverse bleeding outcomes without reduction in risk of stroke. ${ }^{3}$ Aside from renal function, clinicians must also take into consideration bleeding risks, drug interactions, anticipated changes in renal function and patient fragility. ${ }^{3}$ Thus, there is often relevant justification for a prescriber to deviate from a recommended renal-based dose of a DOAC. The $6.9 \%$ rate of 'incorrectly' prescribed doses of rivaroxaban for $A F$ at our institution is much lower than the $35.4 \%$ recently reported by Tellor et al. at another tertiary care community hospital in the Midwest. ${ }^{?}$

\section{Limitations}

One limitation of this study is the fact that it is small, conducted at a single institution, and only assessed one indication (AF) for rivaroxaban. Thus, caution should be utilised when extrapolating these results to the general population or for other DOACs or indications. The retrospective nature of this study also forced investigators to rely on prescriber documentation thus leaving room for bias. As previously mentioned, the number of prescribers for certain disciplines was small and therefore there was no meaningful way to compare their prescribing habits independently. The investigators also relied on patient reporting for adherence and meals. For simplicity, it was only recorded if the patient took the medication with a meal or no meal/snack. Macronutrient content was not assessed. Whether or not the patients received education was also not captured; therefore, conclusions based on counselling practices need to be confirmed with further research.

Finally, because rivaroxaban is a once-daily DOAC for AF, which does not require monitoring like vitamin $K$ antagonist and is generally well tolerated, it has been associated with greater adherence and persistence compared with other anticoagulants. ${ }^{10,11}$ Consequently, prescribers may be more likely to prescribe rivaroxaban patients with documented historical non-adherence. Therefore, non-adherence to the dietary requirements observed in this study may be more attributable to patient characteristics as opposed to lack of patient education by the healthcare providers.

\section{Conclusions}

Though patients prescribed rivaroxaban by a cardiologist in this study were more likely to have received education regarding correct administration of rivaroxaban, there is clearly an education gap that has been supported by other studies. ${ }^{9}$ This highlights the importance of formal systematic education for all patients prescribed rivaroxaban and other DOACs, in the hospital, office and community settings, including information regarding co-administration with a meal. Future studies are also warranted to explore the impact of non-adherence to rivaroxaban dietary requirements on clinical outcomes. $\square$ 
1. Janssen \& Bayer. Rivaroxaban (Xarelto) package insert. Titusville, NJ; 2011.

2. Alamneh EA, Chalmers L, Bereznicki LR. Suboptimal use of oral anticoagulants in atrial fibrillation: has the introduction of direct oral anticoagulants improved prescribing practices? Am I Cardiovasc Drugs. 2016;16:183-200.

3. Yao X, Shah ND, Sangaralingham LR, et al. Non-vitamin K antagonis oral anticoagulant dosing in patients with atrial fibrillation and rena dysfunction. J Am Coll Cardiol. 2017;69:2779-90.

4. Jackevicius CA, Tsadok MA, Essebag V, et al. Early non-persistence with dabigatran and rivaroxaban in patients with atrial fibrillation. Heart. 2017;1:1-18.
5. Stampfuss J, Kubitza D, Becka M, Mueck W. The effect of food on the absorption and pharmacokinetics of rivaroxaban. Int I Clin Pharmacol Ther. 2013;51:549-61.

6. Kubitza D, Becka M, Zuehldorf M, Mueck W. Effect of food, an antacid, and the $\mathrm{H} 2$ antagonist ranitidine on the absorption of antacid, and the $\mathrm{H} 2$ antagonist ranitidine on the absorption of
BAY 59-7939 (rivaroxaban), an oral, direct factor Xa inhibitor, in healthy subjects. J Clin Pharmacol. 2006;46:549-58. 7. Beyer-Westendorf J, Siegert G. Of men and meals. J Thromb Haemost. 2015:13:943-45.

8. Patel MR, Mahaffey KW, Garg J, et al. Rivaroxaban versus warfarin in nonvalvular atrial fibrillation. $N$ Engl I Med. 2011;365:883-91.
9. Tellor KB, Patel S, Armbruster AL, Daly MW. Evaluation of the appropriateness of dosing. Indication and safety of rivaroxapa in a community hospital I Clin Pharm Ther. 2015;40:447-51

10. MCHorny CA, Peterson ED, Laliberte $F$, et al. Comparison of adherence to rivaroxaban versus apixaban among patients with atrial fibrillation. Clin Ther. 2016;38:2477-88.

11. Beyer-Westendorf J, Ehlken B, Evers T. Real-world persistence and adherence to oral anticoagulation for stroke risk reduction in patients with atrial fibrillation. Europace. 2016;18:1150-7. 